\title{
Development of a HPLC-MS/MS Method to Determine 11 Bioactive Compounds in Tongmai Yangxin Pill and Application to a Pharmacokinetic Study in Rats
}

\author{
Jiayuan Shen, Juan Wei, Li Li, Huizi Ouyang, Yanxu Chang $(\mathbb{D}$, \\ Xiaopeng Chen $\mathbb{D}$, and Jun He
}

Tianjin State Key Laboratory of Modern Chinese Medicine, Tianjin University of Traditional Chinese Medicine, Tianjin 300193, China

Correspondence should be addressed to Xiaopeng Chen; xpchen@tjutcm.edu.cn and Jun He; hejun673@163.com

Received 20 August 2018; Accepted 13 September 2018; Published 26 September 2018

Academic Editor: Armando Zarrelli

Copyright (C) 2018 Jiayuan Shen et al. This is an open access article distributed under the Creative Commons Attribution License, which permits unrestricted use, distribution, and reproduction in any medium, provided the original work is properly cited.

A sensitive and reliable HPLC-MS/MS method has been developed and validated for simultaneous determination of eleven bioactive compounds (rhein, emodin, stilbene glycoside, liquiritin, ononin, verbascoside, gallic acid, schisandrin, liquiritigenin, glycyrrhizic acid, and isoliquiritigenin) in rat plasma after oral administration of Tongmai Yangxin Pill. The collected plasma samples were prepared by liquid-liquid extraction with ethyl acetate after acidification. Eleven compounds were separated on a CORTECS $^{\mathrm{TM}} \mathrm{C} 18$ column with mobile phases consisting of $0.1 \%$ formic acid in deionized water and acetonitrile. The flow rate was $0.3 \mathrm{~mL} / \mathrm{min}$. The detection was performed on a tandem mass system with an electrospray ionization (ESI) source in both positive and negative ionization using multiple-reaction monitoring (MRM) mode. The calibration curves were linear over the range of $8-2000 \mathrm{ng} / \mathrm{mL}$ for glycyrrhizic acid; $4-1000 \mathrm{ng} / \mathrm{mL}$ for liquiritin; $0.8-200 \mathrm{ng} / \mathrm{mL}$ for emodin, gallic acid, ononin, schisandrin, and stilbene glycoside; $0.4-100 \mathrm{ng} / \mathrm{mL}$ for isoliquiritigenin, liquiritigenin, rhein, and verbascoside, respectively. The intra- and interday precision of the analytes were less than $9.3 \%$ and $8.5 \%$. The intra- and interday accuracy were in the range of $-14.0 \%$ to $10.3 \%$ and $-6.5 \%$ to $9.6 \%$. Meanwhile, the extraction recovery of the analytes in plasma samples ranged from $85.2 \%$ to $109.1 \%$ and matrix effect from $89.2 \%$ to $113.4 \%$. The developed method was successfully applied to the pharmacokinetics of eleven bioactive compounds in rat plasma after oral administration of Tongmai Yangxin Pill prescription.

\section{Introduction}

Traditional Chinese Medicine (TCM) is a precious treasure of nature. TCMs have been used in clinical for thousands of years and attracting rising attentions due to the treatment of divers diseases successfully with minimum side effects [1-3]. TCM prescription, the most commonly used form in clinical medication, has multicomponents and multitargets [4]. The multicomponents and multitargets are superiority and characteristic of TCM pharmacological actions [5].

Tongmai Yangxin Pill (TMYX) is a traditional Chinese patent medicine documented in Chinese Pharmacopoeia. The prescription was developed from a well-known herb pair "GuiZhi-GanCao" documented in "Shang-Han-Lun" by
Zhongjing Zhang in the Eastern Han Dynasty. The prescription consists of eleven herbs including Radix rehmanniae, Caulis spatholobi, Radix glycyrrhizae, Ramulus cinnamomi, Radix ophiopogonis, Radix polygoni multiflori preparata, Asini corii colla, Fructus schisandrae, Radix codonopsis, Capapax et Plastrum testudinis, and Fructus jujubae [6]. TMYX has been used to treat coronary heart disease, arrhythmia, chest pain, and angina for several decades [7-9]. Modern pharmacological studies show that TMYX has a significant effect on heart disease. Four active flavonoid compounds (glyasperin A, glycycoumarin, licorisoflavan A, and licoisoflavone A) from TMYX were found to have satisfactory biological activity and promote proliferation and angiogenesis of human umbilical vein endothelial cells in zebrafish. [10]. Meanwhile, Liu et al. reported that five fractions of TMYX were found 
to exert antiepithelial-mesenchymal transition activity [11]. Tao's results indicated that six active ingredients with high $R$ values (gomisin $\mathrm{D}$, schisandrin, glycyrrhizic acid, stilbene glycoside, formononetin, and ononin) exert antiinflammatory effects in a dose-dependent manner [12].

The pharmacological effects of TMYX are based on the diverse chemical composition. Chen reported 80 compounds were identified or surmised using HPLC-MS, including 23 flavones and their glucuronides, 6 phenethyl alcohol glycosides, 20 triterpene saponins, 15 lignans, and 18 other compounds [13]. Fan characterized 40 absorbed bioactive components after oral administration of TMYX in rat serum by UPLC/Q-TOF-MS [14]. The 40 components including 2 from Radix rehmanniae, 10 from Radix codonopsis, 2 from Radix ophiopogonis, 2 from Ramulus cinnamomi, 19 from Radix glycyrrhizae, 2 from Radix polygoni multiflori preparata, 5 from Caulis spatholobi, 1 from Fructus jujubae, and 1 from Fructus schisandrae, some of which are overlapped. Most of the ingredients exert anti-inflammatory and antioxidation effects, furthermore exerting the cardiovascular protective effect. The team also determines the concentrations of liquiritin, liquiritigenin, isoliquiritigenin, glycyrrhizic acid, and glycyrrhetinic acid in rat plasma following oral administration of Radix glycyrrhizae or the combination of Radix glycyrrhizae and Ramulus cinnamomi by HPLC-UV. However, there is no publication that reports the pharmacokinetic study of TMYX.

Pharmacokinetics (PK) of TCMs is a branch of the pharmacology of TCMs. PK of TCMs focuses on quantitatively studies the laws of drug absorption, distribution, metabolism, and excretion in a living organism. PK study of multicomponents of TCM prescription has been one of the important research aspects of modernization of TCMs [1]. The PK data could elucidate the substance basis and reveal the scientific connotation of TCMs. It also plays an important role in the creation of new Chinese medicines, the improvement of dosage forms, and the mechanism of formulation mechanism. In the present study, a reliable and sensitive HPLC-MS/MS method was first developed and applied to the pharmacokinetic study of 11 bioactive components including rhein, emodin, stilbene glycoside, liquiritin, ononin, verbascoside, gallic acid, schisandrin, liquiritigenin, glycyrrhizic acid and isoliquiritigenin in rats after oral administration of TMYX. The pharmacokinetic characteristics of the main chemical components of TMYX in rats were revealed, which would provide a theoretical basis for use of TMYX in clinical.

\section{Experimental}

2.1. Chemicals, Reagents, and Materials. Methanol (chromatographic purity) and acetonitrile (chromatographic purity) were purchased from Merck Co., Ltd. Formic acid (chromatographic purity) was obtained from ROE Co., Ltd. Ultra-pure water was prepared with a Milli-Q water purification system (Millipore, Milford, MA, USA). Rhein, emodin, stilbene glycoside, liquiritin, ononin, verbascoside, gallic acid, schisandrin, liquiritigenin, glycyrrhizic acid, isoliquiritigenin, and icariin were purchased from Chengdu Must Bio-Technology Co., Ltd. (Chengdu, China). TMYX were supplied by Tianjin Zhongxin Pharmaceutical Group Co., Ltd.
2.2. Chromatographic and Mass Spectrometry Conditions. The HPLC-MS/MS system consists of an Agilent 1200 high-performance liquid chromatography coupled with an Aglient 6430 series triple quadrupole mass spectrometer with an electrospray ionization (ESI) source. The chromatographic separation was achieved on a CORTECS C18 column $(4.6 \mathrm{~mm}$ $\times 150 \mathrm{~mm}, 2.7 \mu \mathrm{m})$, and the column temperature was kept at $30^{\circ} \mathrm{C}$. Mobile phases which consisted of $0.1 \%$ formic acid in water (A) and acetonitrile (B) were used in the following gradient elution method: $0-10 \mathrm{~min}, 10 \%-85 \% \mathrm{~B} ; 10-13 \mathrm{~min}$, 85\%-95\% B; $13-19 \mathrm{~min}, 95 \%-95 \% \mathrm{~B}$. The flow rate was set at $0.3 \mathrm{~mL} / \mathrm{min}$, and the injection volume was $10 \mu \mathrm{L}$. All data were analyzed by Mass Hunter workstation software (Agilent Technologies, USA).

The mass spectrometer was carried out in both positive and negative ionization multiple-reaction monitoring (MRM) mode. The source parameters were as follows: the capillary voltage set at $300 \mathrm{~V}$ for positive ionization mode and $-300 \mathrm{~V}$ for negative ionization mode, the drying gas temperature was $320^{\circ} \mathrm{C}$, the flow was $11 \mathrm{~L} / \mathrm{min}$, and nebulizing gas pressure was $30 \mathrm{psi}$. The precursor and production of the ingredients and MRM parameters were displayed in Table 1.

2.3. TMYX Extract Preparation. TMYX extract was prepared as follows: A total of $100 \mathrm{~g}$ TMYX powder was accurately weighed and extracted twice under heat reflux by four times amounts of $60 \%$ ethanol (v/v) for $1 \mathrm{~h}$ per time. After that, the extraction solutions were filtered and mixed. The mixed solution was concentrated by evaporation under reduced pressure. And then the dried extracts was crushed to powder and kept in a desiccator until analysis. The extracts contains rhein, emodin, stilbene glycoside, liquiritin, ononin, verbascoside, gallic acid, schisandrin, liquiritigenin, glycyrrhizic acid, and isoliquiritigenin 0.4, 46.3, 231.6, 270.5, 161.1, 27.8, $71.8,65.2,16.9,519.7$, and $9.6 \mu \mathrm{g} / \mathrm{g}$, respectively. The structures of the compounds in the study were shown in Figure 1.

2.4. Calibration Solutions and Quality Control Samples Preparation. To make the stock solution, rhein, emodin, stilbene glycoside, liquiritin, ononin, verbascoside, gallic acid, schisandrin, liquiritigenin, glycyrrhizic acid, isoliquiritigenin, and icariin (internal standard solution) were weighed separately and diluted with methanol to a final concentration of $1 \mathrm{mg} / \mathrm{mL}$. The mixed standard solution was obtained by mixed appropriate volume of eleven stock solutions and diluted with methanol.

The calibration solutions were prepared by spiking the $20 \mu \mathrm{L}$ of the mixture standard solution and $20 \mu \mathrm{L}$ of IS into $100 \mu \mathrm{L}$ blank rat plasma. The final concentrations of the series analytes were at the range of $8-2000 \mathrm{ng} / \mathrm{mL}$ for glycyrrhizic acid; 4-1000 ng/mL for liquiritin; 0.8-200 ng/mL for emodin, gallic acid, ononin, schisandrin, and stilbene glycoside; and 0.4-100 ng/mL for isoliquiritigenin, liquiritigenin, rhein, and verbascoside.

Quality control (QC) samples at three concentrations (low, medium, and high concentration) were made up of appropriate mixed standard solutions with blank blood sample as calibration solutions to meet the required concentrations. All the solutions were kept at $4^{\circ} \mathrm{C}$. 
TABLE 1: Mass spectra properties of 11 analytes and IS.

\begin{tabular}{|c|c|c|c|c|}
\hline Compounds & Precursor Ion $(\mathrm{m} / z)$ & Product Ion $(m / z)$ & Frag. (V) & C.E. (V) \\
\hline rhein & 238.9 & 211.0 & 140 & -10 \\
\hline emodin & 269.0 & 225.0 & 145 & -20 \\
\hline stilbene glycoside & 405.2 & 242.8 & 145 & -13 \\
\hline liquiritin & 417.1 & 255.0 & 145 & -13 \\
\hline ononin & 431.2 & 269.1 & 10 & 13 \\
\hline verbascoside & 623.0 & 161.1 & 116 & -33 \\
\hline gallic acid & 169.0 & 125.1 & 123 & -12 \\
\hline schisandrin & 433.3 & 384.2 & 100 & 14 \\
\hline liquiritigenin & 255.3 & 119.1 & 121 & -24 \\
\hline glycyrrhizic acid & 821.1 & 350.5 & 125 & -40 \\
\hline isoliquiritigenin & 255.1 & 118.9 & 106 & -23 \\
\hline icariin (IS) & 721.0 & 513.2 & 145 & -10 \\
\hline
\end{tabular}

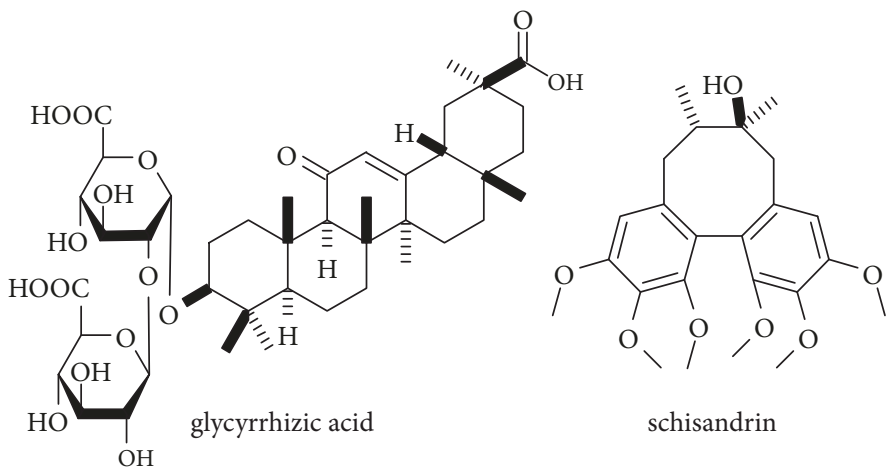<smiles>CCCCCCCCCCOC(=O)/C=C/c1ccc(O)c(O)c1</smiles><smiles>Cc1cc(O)c2c(c1)C(=O)c1cc(O)cc(O)c1C2=O</smiles><smiles>O=C(O)c1cc(O)c2c(c1)C(=O)c1cccc(O)c1C2=O</smiles><smiles>O=C1C[C@H](c2ccc(O)cc2)Oc2cc(O)ccc21</smiles>

liquiritigenin<smiles>O=C(/C=C/c1ccc(O)cc1)c1ccc(O)cc1O</smiles>

isoliquiritigenin<smiles></smiles>

stilbene glycoside<smiles>O=C1C[C@@H](c2ccc(O)cc2)Oc2cc(O)ccc21</smiles>

liquiritin

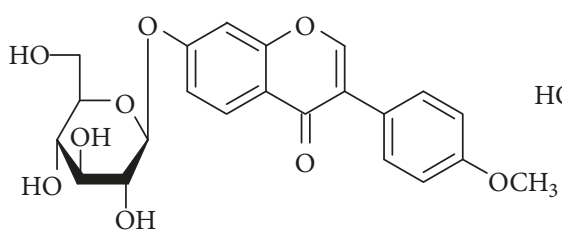

ononin<smiles>O=C(O)c1cc(O)c(O)c(O)c1</smiles>

gallic acid

Figure 1: Chemical structures of eleven components. 
TABLE 2: Calibration curves, correlation coefficients, linear ranges, and LLOQ of the analytes.

\begin{tabular}{|c|c|c|c|c|}
\hline Compounds & Calibration curves & Correlation coefficients (r) & Linear range $(\mathrm{ng} / \mathrm{mL})$ & LLOQ (ng/mL) \\
\hline rhein & $y=0.0954 x+1.5911$ & 0.9966 & $0.4-100$ & 0.4 \\
\hline emodin & $y=1.2189 x+0.0135$ & 0.9906 & $0.8-200$ & 0.8 \\
\hline stilbene glycoside & $y=6.6409 x+0.0293$ & 0.9991 & $0.8-200$ & 0.8 \\
\hline gallic acid & $y=0.9987 x+0.0104$ & 0.9987 & $0.8-200$ & 0.8 \\
\hline ononin & $y=5.4632 x+0.0058$ & 0.9995 & $0.8-200$ & 0.8 \\
\hline verbascoside & $y=0.3514 x+1.9984$ & 0.9965 & $0.4-100$ & 0.4 \\
\hline liquiritin & $y=1.1970 x+0.0085$ & 0.9965 & $4-1000$ & 4 \\
\hline schisandrin & $y=2.5769 x+0.0020$ & 0.9980 & $0.8-200$ & 0.8 \\
\hline liquiritigenin & $y=2.2148 x+0.0140$ & 0.9997 & $0.4-100$ & 0.4 \\
\hline glycyrrhizic acid & $y=0.0917 x-0.0028$ & 0.9982 & $8-2000$ & 8 \\
\hline isoliquiritigenin & $y=3.1496 x+0.0064$ & 0.9986 & $0.4-100$ & 0.4 \\
\hline
\end{tabular}

2.5. Plasma Sample Preparation. The plasma $(100 \mu \mathrm{L})$ was spiked with $20 \mu \mathrm{L}$ of methanol, $20 \mu \mathrm{L}$ of the IS (icariin, $1 \mu \mathrm{g}$ / $\mathrm{mL}$ ), and $20 \mu \mathrm{L}$ of formic acid and then vortex-mixed. The mixture was extracted with $800 \mu \mathrm{L}$ of ethyl acetate by vortex mixing for $5 \mathrm{~min}$ at room temperature. After centrifugation at $14,000 \mathrm{rpm}$ for $10 \mathrm{~min}$, the supernatant was collected to a clean tube and evaporated to dryness under a nitrogen stream. The residue was reconstituted in $50 \mu \mathrm{L}$ of $50 \%$ methanol, vortexed for $5 \mathrm{~min}$, and centrifuged at $14,000 \mathrm{rpm}$ for $10 \mathrm{~min}$. Finally, $10 \mu \mathrm{L}$ supernatant was injected into the LC-MS/MS system for analysis.

\subsection{Method Validation}

2.6.1. Specificity. The specificity was assessed by analyzing blank blood samples from six different rats. Each plasma sample was evaluated for endogenous interference using the suggested extraction program and LC-MS/MS conditions above.

2.6.2. Linearity and $L L O Q$. The linearity was achieved by assaying blank rat plasma with a serious of the mixed standard solution and IS in duplicate over 3 consecutive days. The calibration curves were plotted by the peak-area ratios (y) of analyte against internal standard versus the nominal concentration $(\mathrm{x})$. The weight factor is $1 / x$. The lower limit of quantification (LLOQ) was evaluated according to the base line noise, defining a signal-to-noise ratio of about 10 .

2.6.3. Precision and Accuracy. The precision and accuracy were assessed by determining QC samples at low, medium, and high concentration levels $(20,200$, and $2000 \mathrm{ng} / \mathrm{mL}$ for glycyrrhizic acid; 10,100 , and $1000 \mathrm{ng} / \mathrm{mL}$ for liquiritin; 2, 20, and $200 \mathrm{ng} / \mathrm{mL}$ for emodin, gallic acid, ononin, schisandrin, and stilbene glycoside; 1,10 , and $100 \mathrm{ng} / \mathrm{mL}$ for isoliquiritigenin, liquiritigenin, rhein, and verbascoside). All concentration levels were measured in six replicates. The precision and accuracy were tested once a day and repeated for 3 consecutive days with the standard calibration curve. Intra- and interday precision were defined as the relative standard deviation (RSD), while the accuracy was determined by the relative error (RE \%).

2.6.4. Extraction Recovery and Matrix Effect. The extraction recovery of eleven analytes at three concentration levels and IS were assayed by comparing the peak areas obtained from extracted samples with those of the postextracted samples. The matrix effect of the samples and IS were estimated by the peak-area ratios of the analytes in postextracted spiked samples to those obtained from unextracted samples. Both the extraction recovery and matrix effect were tested in six parallels.

2.6.5. Stability. The stability of analytes in plasma samples was determined by analyzing QC samples of three concentration levels at different conditions: stored at autosampler for $12 \mathrm{~h}$, at room temperature for $6 \mathrm{~h}$, under three freeze-thaw cycles and stored at $-70^{\circ} \mathrm{C}$ for 14 days. All stability studies were measured in six replicates.

2.7. Pharmacokinetic Study. Male Sprague-Dawley rats (230$250 \mathrm{~g}$ ) were obtained from Beijing HFK Experimental Animal Technology Co., Ltd. The rats were maintained under the control environmental conditions and they fasted for $12 \mathrm{~h}$ with free access to water before the experiments. TMYX extracts were dissolved in CMC-Na and administrated orally to rats at $8.3 \mathrm{~g} / \mathrm{kg}$. The blood samples $(200 \mu \mathrm{L})$ were collected from the rat fossa orbitalis vein at $0,0.03,0.083,0.17,0.25,0.5$, $1,2,4,6,8,10,12,24,36$, and $48 \mathrm{~h}$ after oral administration to heparinized tubes. And then the blood samples were centrifuged at 7,000 rpm for $10 \mathrm{~min}$ to get the plasma sample immediately. Finally, the plasma obtained was stored at $-70^{\circ} \mathrm{C}$ until analysis. Pharmacokinetic parameters were calculated by the computer program "Drug and Statistics 2.0" (DAS 2.0) (Medical College of Wannan, China).

\section{Result and Discussion}

3.1. HPLC-MS/MS Method. Several mobile phases were tested. Comparing with a gradient mobile phase system 


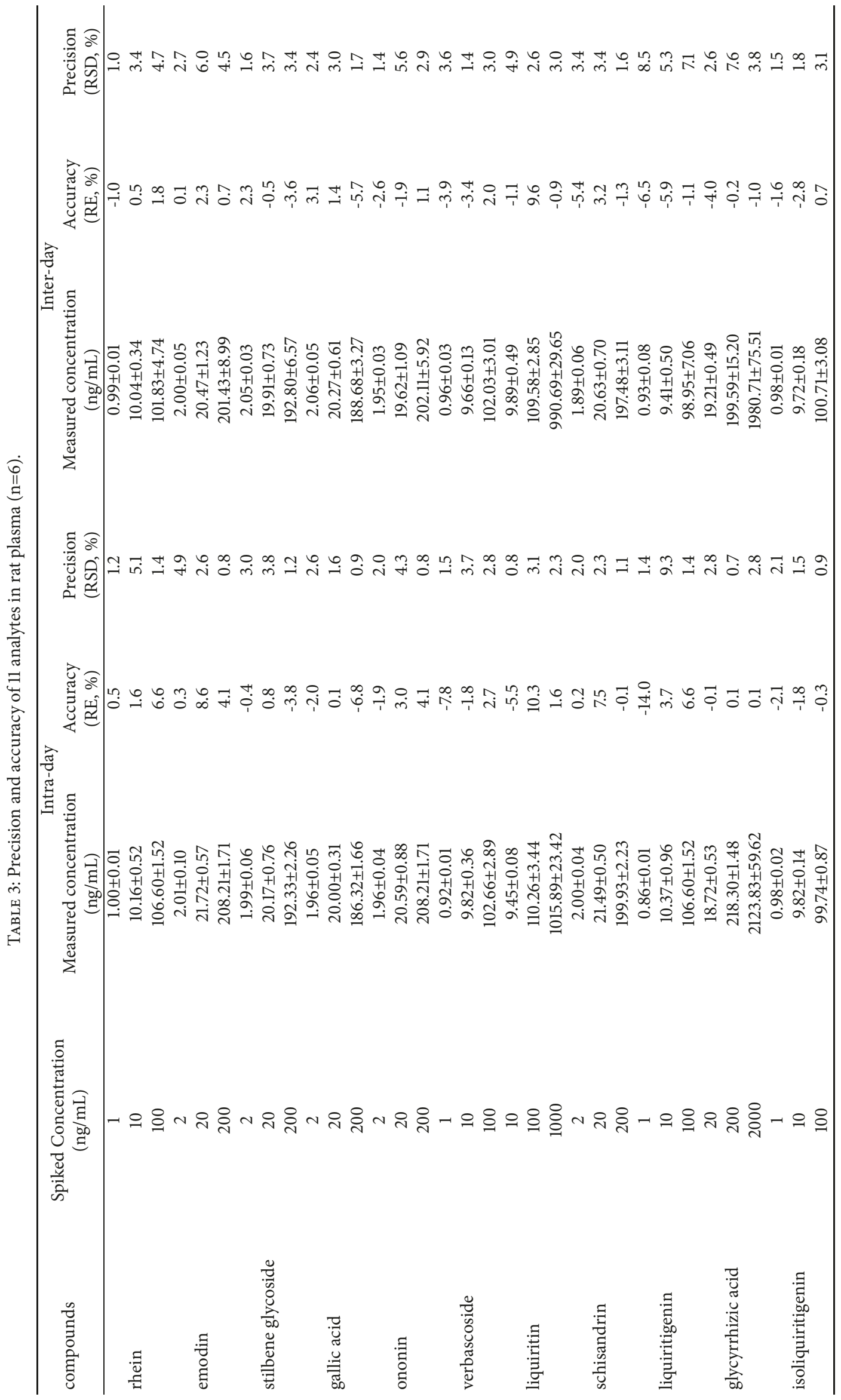


TABLE 4: Extraction recoveries and matrix effects of the analytes $(n=6)$.

\begin{tabular}{|c|c|c|c|c|c|}
\hline Compounds & Spiked concentration $(\mathrm{ng} / \mathrm{mL})$ & Extraction recovery (\%) & RSD (\%) & Matrix effect (\%) & RSD (\%) \\
\hline \multirow{3}{*}{ rhein } & 1 & $96.2 \pm 7.4$ & 7.7 & $104.1 \pm 5.6$ & 5.4 \\
\hline & 10 & $99.3 \pm 3.9$ & 3.9 & $100.1 \pm 2.7$ & 2.7 \\
\hline & 100 & $101.4 \pm 5.2$ & 5.1 & $92.6 \pm 3.7$ & 4.0 \\
\hline \multirow{3}{*}{ emodin } & 2 & $98.8 \pm 3.4$ & 3.5 & $107.7 \pm 2.6$ & 2.4 \\
\hline & 20 & $96.2 \pm 2.5$ & 2.6 & $103.6 \pm 0.8$ & 0.8 \\
\hline & 200 & $86.1 \pm 4.2$ & 4.9 & $104.8 \pm 2.9$ & 2.8 \\
\hline \multirow{3}{*}{ stilbene glycoside } & 2 & $98.9 \pm 1.9$ & 1.9 & $100.7 \pm 2.2$ & 2.2 \\
\hline & 20 & $99.3 \pm 4.0$ & 4.0 & $94.6 \pm 2.2$ & 2.3 \\
\hline & 200 & $96.7 \pm 5.6$ & 5.8 & $98.7 \pm 0.8$ & 0.9 \\
\hline \multirow{3}{*}{ gallic acid } & 2 & $99.1 \pm 3.1$ & 3.2 & $110.4 \pm 5.8$ & 5.2 \\
\hline & 20 & $100.9 \pm 2.5$ & 2.5 & $98.3 \pm 2.0$ & 2.0 \\
\hline & 200 & $85.2 \pm 3.0$ & 3.5 & $108.1 \pm 1.3$ & 1.2 \\
\hline \multirow{3}{*}{ ononin } & 2 & $91.8 \pm 2.7$ & 2.9 & $98.6 \pm 2.8$ & 2.8 \\
\hline & 20 & $89.0 \pm 3.8$ & 4.3 & $89.9 \pm 0.7$ & 0.8 \\
\hline & 200 & $93.1 \pm 5.2$ & 5.6 & $91.7 \pm 0.9$ & 1.0 \\
\hline \multirow{3}{*}{ verbascoside } & 1 & $93.1 \pm 4.0$ & 4.3 & $103.6 \pm 3.3$ & 3.1 \\
\hline & 10 & $97.0 \pm 4.7$ & 4.8 & $100.0 \pm 2.1$ & 2.1 \\
\hline & 100 & $92.5 \pm 6.1$ & 6.6 & $98.9 \pm 2.3$ & 2.4 \\
\hline \multirow{3}{*}{ liquiritin } & 10 & $105.7 \pm 4.5$ & 4.3 & $98.8 \pm 1.2$ & 1.2 \\
\hline & 100 & $109.1 \pm 4.0$ & 3.6 & $100.1 \pm 6.4$ & 6.4 \\
\hline & 1000 & $99.5 \pm 7.6$ & 7.7 & $95.2 \pm 5.5$ & 5.8 \\
\hline \multirow{3}{*}{ schisandrin } & 2 & $98.8 \pm 4.1$ & 4.2 & $96.8 \pm 2.6$ & 2.7 \\
\hline & 20 & $106.3 \pm 1.1$ & 1.0 & $93.0 \pm 1.7$ & 1.8 \\
\hline & 200 & $101.0 \pm 6.0$ & 5.9 & $90.4 \pm 0.7$ & 0.8 \\
\hline \multirow{3}{*}{ liquiritigenin } & 1 & $99.7 \pm 3.5$ & 3.6 & $113.4 \pm 2.8$ & 2.5 \\
\hline & 10 & $99.5 \pm 2.7$ & 2.7 & $89.2 \pm 1.3$ & 1.5 \\
\hline & 100 & $96.3 \pm 3.4$ & 3.5 & $99.8 \pm 1.8$ & 1.8 \\
\hline \multirow{3}{*}{ glycyrrhizic acid } & 20 & $100.9 \pm 7.5$ & 7.4 & $101.6 \pm 6.8$ & 6.7 \\
\hline & 200 & $97.5 \pm 2.1$ & 2.1 & $102.5 \pm 1.3$ & 1.3 \\
\hline & 2000 & $87.6 \pm 1.6$ & 1.8 & $103.7 \pm 2.1$ & 2.0 \\
\hline \multirow{3}{*}{ isoliquiritigenin } & 1 & $98.0 \pm 5.5$ & 5.6 & $105.73 \pm 2.2$ & 2.1 \\
\hline & 10 & $96.5 \pm 2.0$ & 2.1 & $98.0 \pm 3.6$ & 3.6 \\
\hline & 100 & $85.9 \pm 5.7$ & 6.7 & $97.3 \pm 3.1$ & 3.2 \\
\hline
\end{tabular}

with acetonitrile-water or methanol-water, the mobile phase contains $0.1 \%$ formic acid in water could improve the peak shape and increase the signal response of the analytes. Thus, we chose the water containing $0.1 \%$ formic acid as the mobile phase.

The standard solutions of analytes and internal standard were injected into the mass spectrometer, respectively. The ononin and schisandrin were tested in positive ion mode, while others in negative. The optimized precursorto-production transitions were monitored at $238.9 \longrightarrow 211.0$ for rhein, $269.0 \longrightarrow 225.0$ for emodin, $405.2 \longrightarrow 242.8$ for stilbene glycoside, $417.1 \longrightarrow 255.0$ for liquiritin, $431.2 \longrightarrow 269.1$ for ononin, $623.0 \longrightarrow 161.1$ for verbascoside, $169.0 \longrightarrow 125.1$ for gallic acid, $433.3 \longrightarrow 384.2$ for schisandrin, $255.3 \longrightarrow 119.1$ for liquiritigenin, $821.1 \longrightarrow 350.5$ for glycyrrhizic acid, $255.1 \longrightarrow 118.9$ for isoliquiritigenin, and $721.0 \longrightarrow 513.2$ for IS. All data were shown in Table 1.
3.2. Sample Preparation. In the experiment, we tested two methods to dispose the plasma sample including liquidliquid extraction (LLE) and protein precipitation (PPT). The results show that both the recovery and matrix effects of the methods meet the requirements for the determination of biological samples and the endogenous substances do not interfere with the analysis. However, the method of PPT showed lower extraction efficiency and higher matrix effect relatively. Consequently, we selected liquid-liquid extraction (LLE) with ethyl acetate to prepare the sample.

\subsection{Method Validation}

3.3.1. Specificity. The specificity was estimated by comparing chromatograms of blank blood samples from six different rats with blood samples containing analytes. The representative chromatograms of blank blood sample, blank blood 


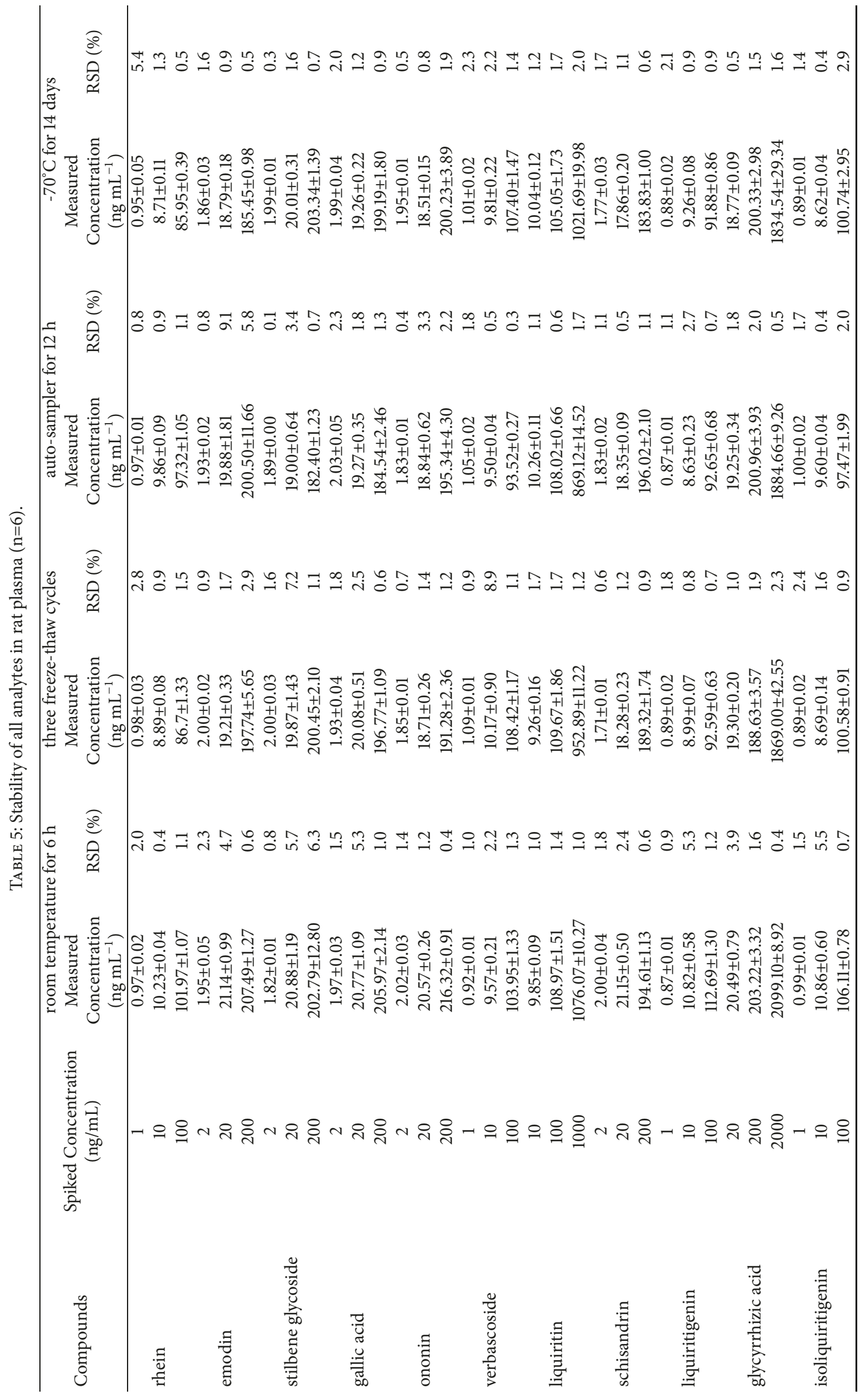


TABLE 6: Pharmacokinetic parameters of 11 analytes after oral administration of TMYX extract $(n=6)$.

\begin{tabular}{|c|c|c|c|c|c|c|c|c|}
\hline Compounds & $T_{\operatorname{maxl}}(\mathrm{h})$ & $T_{\max 2}(\mathrm{~h})$ & $C_{\operatorname{maxl}}(\mathrm{ng} / \mathrm{mL})$ & $C_{\max 2}(\mathrm{ng} / \mathrm{mL})$ & $t_{1 / 2}(\mathrm{~h})$ & $K_{e}(1 / \mathrm{h})$ & $A U C_{(0-t n)}(\mathrm{h} \cdot \mathrm{ng} / \mathrm{mL})$ & $A U C_{(0-\infty)}(\mathrm{h} \cdot \mathrm{ng} / \mathrm{mL})$ \\
\hline rhein & $0.36 \pm 0.31$ & & $46.3 \pm 15.6$ & & $2.84 \pm 2.13$ & $0.56 \pm 0.50$ & $145.0 \pm 52.6$ & $152.1 \pm 61.3$ \\
\hline emodin & $0.32 \pm 0.14$ & & $88.0 \pm 37.5$ & & $4.21 \pm 2.63$ & $0.39 \pm 0.28$ & $475.5 \pm 121.0$ & $561.3 \pm 192.3$ \\
\hline stilbene glycoside & $0.50 \pm 0.31$ & & $99.1 \pm 27.1$ & & $1.99 \pm 1.31$ & $0.45 \pm 0.24$ & $283.8 \pm 189.1$ & $297.5 \pm 185.3$ \\
\hline liquiritin & $0.50 \pm 0.46$ & & $199.9 \pm 120.2$ & & $2.16 \pm 1.44$ & $0.77 \pm 0.54$ & $637.2 \pm 220.2$ & $651.1 \pm 219.5$ \\
\hline ononin & $0.46 \pm 0.10$ & & $14.4 \pm 6.8$ & & $2.85 \pm 1.40$ & $0.37 \pm 0.25$ & $58.2 \pm 20.2$ & $69.4 \pm 28.5$ \\
\hline verbascoside & $0.37 \pm 0.18$ & & $23.4 \pm 8.7$ & & $1.53 \pm 0.67$ & $0.56 \pm 0.31$ & $51.4 \pm 29.8$ & $61.0 \pm 29.8$ \\
\hline gallic acid & $0.75 \pm 0.67$ & & $56.9 \pm 42.8$ & & $3.01 \pm 1.35$ & $0.27 \pm 0.12$ & $214.7 \pm 136.7$ & $238.4 \pm 172.8$ \\
\hline schisandrin & $1.00 \pm 0.92$ & & $92.6 \pm 53.0$ & & $2.73 \pm 1.54$ & $0.34 \pm 0.19$ & $410.8 \pm 238.9$ & $473.4 \pm 321.2$ \\
\hline liquiritigenin & $0.20 \pm 0.07$ & $7.20 \pm 3.35$ & $37.8 \pm 24.1$ & $21.0 \pm 15.3$ & $12.70 \pm 7.04$ & $0.50 \pm 0.34$ & $314.8 \pm 129.8$ & $332.6 \pm 142.1$ \\
\hline glycyrrhizic acid & $2.13 \pm 1.44$ & $17.67 \pm 10.31$ & $351.7 \pm 347.4$ & $476.1 \pm 146.0$ & $18.19 \pm 9.61$ & $0.05 \pm 0.03$ & $12743.5 \pm 5058.2$ & $17327.3 \pm 10967.3$ \\
\hline isoliquiritigenin & $0.28 \pm 0.11$ & $7.00 \pm 2.45$ & $41.6 \pm 24.2$ & $24.9 \pm 13.5$ & $13.46 \pm 8.33$ & $0.07 \pm 0.04$ & $410.0 \pm 130.7$ & $436.2 \pm 154.1$ \\
\hline
\end{tabular}

sample containing eleven analytes and IS, and plasma sample obtained from a rat after oral administration of TMYX extracts were accessed. The retention time of rhein, emodin, stilbene glycoside, liquiritin, ononin, verbascoside, gallic acid, schisandrin, liquiritigenin, glycyrrhizic acid, isoliquiritigenin, and IS were 15.93, 17.63, 11.34, 11.31, 12.21, 11.01, 6.48, $16.41,13.21,13.34,14.41$, and $12.19 \mathrm{~min}$, respectively. Based on the chromatograms, endogenous substances in plasma samples do not interfere with the determination of analytes and IS. The chromatograms were shown in Figure 2.

3.3.2. Linearity and Sensitivity. The results of calibration curves, linear ranges, correlation coefficients, and LLOQs were displayed in Table 2. The plasma calibration curves were constructed within the range of $8-2000 \mathrm{ng} / \mathrm{mL}$ for glycyrrhizic acid; $4-1000 \mathrm{ng} / \mathrm{mL}$ for liquiritin; $0.8-200 \mathrm{ng} / \mathrm{mL}$ for emodin, gallic acid, ononin, schisandrin, and stilbene glycoside; $0.4-100 \mathrm{ng} / \mathrm{mL}$ for isoliquiritigenin, liquiritigenin, rhein, and verbascoside.

The LLOQs for 11 analytes in plasma sample were less than $8 \mathrm{ng} / \mathrm{mL}$, which are sensitive enough for the pharmacokinetic studies.

3.3.3. Precision and Accuracy. In this assay, the intra- and interday precision and accuracy were analyzed at three concentration levels in six replicates. The data were displayed in Table 3. The RSD of intra- and interday precision were between 0.7 and $9.3 \%$. The RE of accuracy was within $\pm 14.0 \%$. The results suggest that the method is accurate and repeatable for analysis of all analytes in rat plasma.

3.3.4. Extraction Recovery and Matrix Effect. All data of the extraction recovery and matrix effect were summarized in Table 4. The extraction recovery of 11 ingredients at three concentration levels were in the scope of $85.2-109.1 \%$. The matrix effects of all analytes ranged from 89.2 to $113.4 \%$. The data which manifests the procedure of the experiment is efficient and the matrix effects could be ignored.

3.3.5. Stability. To investigate the stability of analytes, QC samples of three concentrations levels under the different storage conditions were tested, including stored at autosampler for $12 \mathrm{~h}$ after preparation, at room temperature for $6 \mathrm{~h}$, at three freeze-thaw cycles, and at $-70^{\circ} \mathrm{C}$ for 14 days. As shown in Table 5, the results suggest that the analytes are stable in the above conditions.

3.4. Pharmacokinetic Study. The validated LC-MS/MS method was applied to the pharmacokinetic study of the eleven analytes in rat blood sample after oral administration of TMYX at a single dose of $8.3 \mathrm{~g} / \mathrm{kg}$. The major pharmacokinetic parameters were demonstrated in Table 6 . And the mean plasma concentration-time profiles of the eleven active ingredients were shown in Figure 3.

The $T_{\max }$ of rhein, emodin, stilbene glycoside, liquiritin, ononin, verbascoside, gallic acid, and schisandrin are $0.36 \pm 0.31 \mathrm{~h}, 0.32 \pm 0.14 \mathrm{~h}, 0.50 \pm 0.31 \mathrm{~h}, 0.50 \pm 0.46 \mathrm{~h}, 0.46 \pm$ $0.10 \mathrm{~h}, 0.37 \pm 0.18 \mathrm{~h}, 0.75 \pm 0.67 \mathrm{~h}$, and $1.00 \pm 0.92 \mathrm{~h}$, respectively. The $T_{\max }$ shows that eight ingredients are absorbed rapidly. The double peaks are detected in the mean plasma concentration-time profiles for liquiritigenin, glycyrrhizic acid, and isoliquiritigenin. The first peak of three ingredients appeared at $0.20 \mathrm{~h}, 2.13 \mathrm{~h}$, and $0.28 \mathrm{~h}$ and the second peak at $7.20 \mathrm{~h}, 17.67 \mathrm{~h}$, and $7.00 \mathrm{~h}$, respectively. It might result from hepatoenteral circulation. One peak plasma concentrationtime profiles of liquiritigenin, isoliquiritigenin, and glycyrrhizic acid were reported in rat plasma following oral administration of Radix glycyrrhizae or the combination of Radix glycyrrhizae and Ramulus cinnamomi [8]. The differences may come from the effects of other herbs in TMYX which require further experiment. Besides double peaks, the half-life of elimination $\left(t_{1 / 2}\right)$ of the rhein, emodin, gallic acid, glycyrrhizin, stilbene glycoside, verbascoside, formononetin, and schisandrin range from $1.53 \mathrm{~h}$ to $4.21 \mathrm{~h}$, which suggests that these eight analytes in rat blood sample are eliminated fleetly after oral administration. The similar pharmacokinetic trend of schisandrin was reported after oral administration of Fructus schisandrae aqueous extract [15]. It was also reported that one peak was observed for emodin and gallic acid in rat dosed processed Radix polygoni multiflori [16]. The $t_{1 / 2}$ of the liquiritigenin, glycyrrhizic acid, and isoliquiritigenin are $12.70 \mathrm{~h}, 18.19 \mathrm{~h}$, and $13.46 \mathrm{~h}$, which 

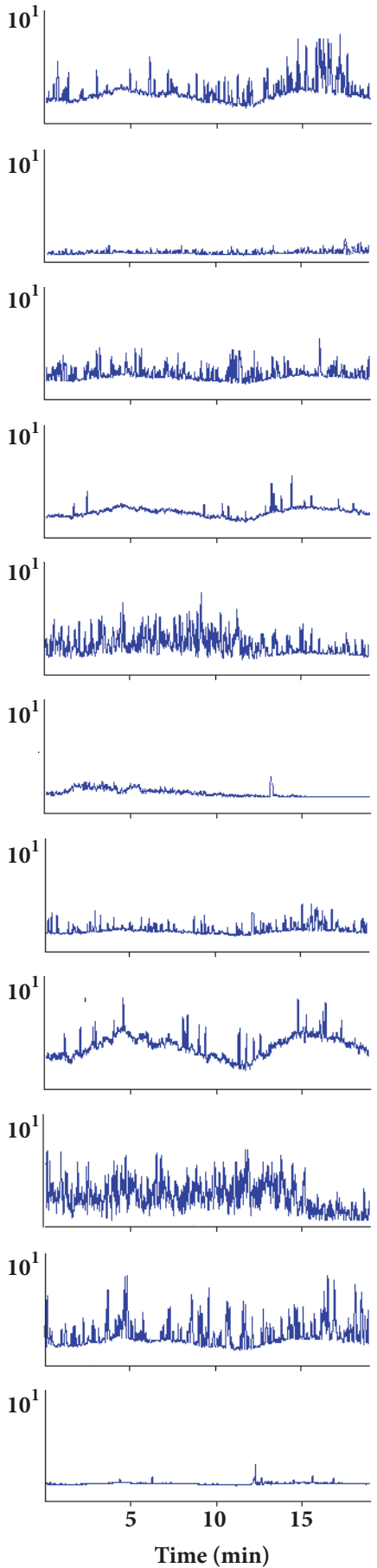

(a)
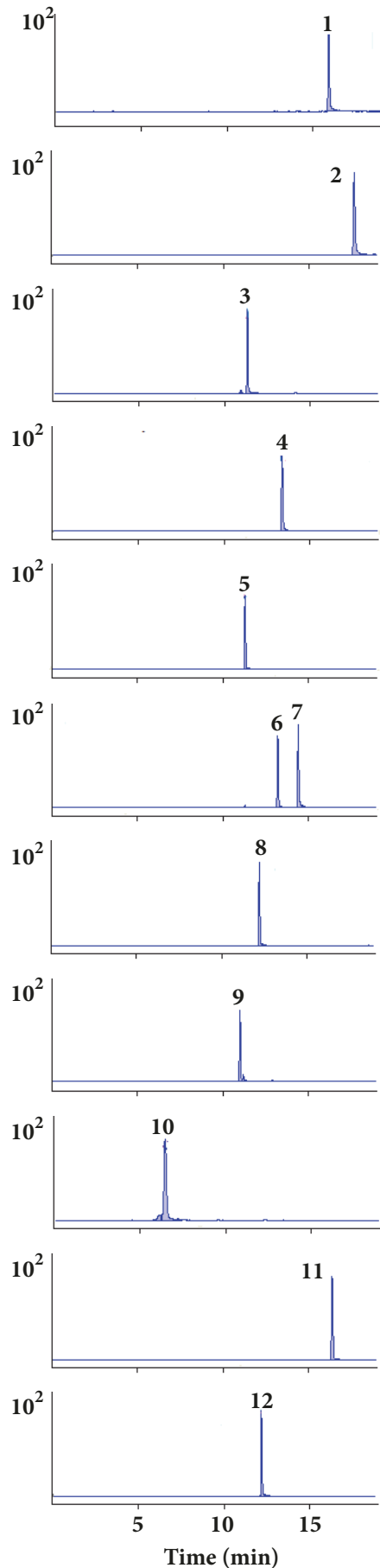

(b)
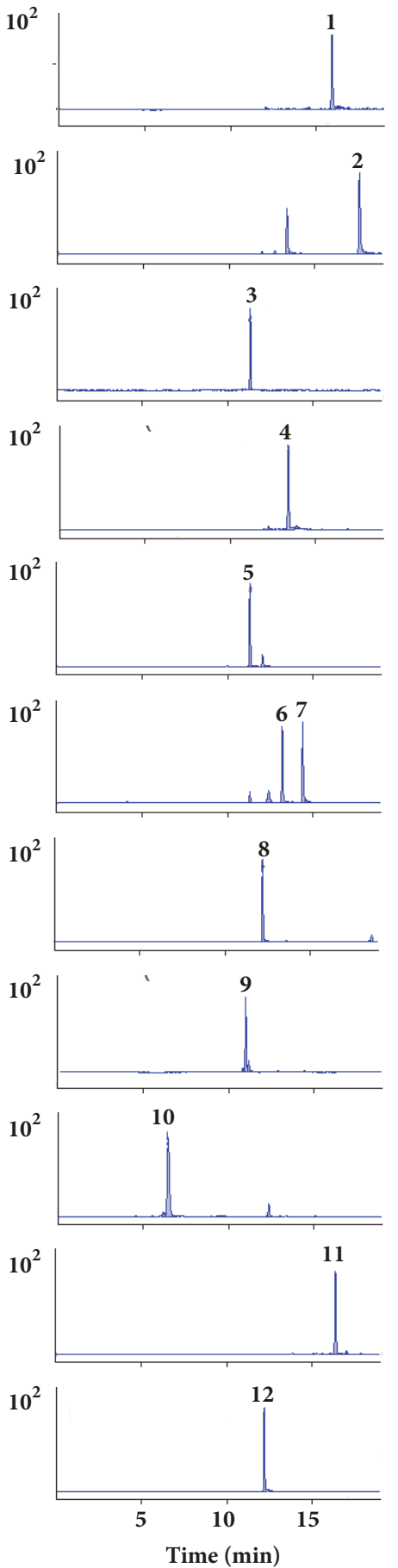

(c)

FIGURE 2: MRM chromatograms of eleven analytes. rhein (1), emodin (2), stilbene glycoside (3), glycyrrhizic acid (4), liquiritin (5), liquiritigenin (6), isoliquiritigenin (7), ononin (8), verbascoside (9), gallic acid (10), schisandrin (11), and IS (12). (a) Blank plasma; (b) blank plasma spiked with the analytes and IS; (c) plasma sample after oral administration of TMYX extract. 

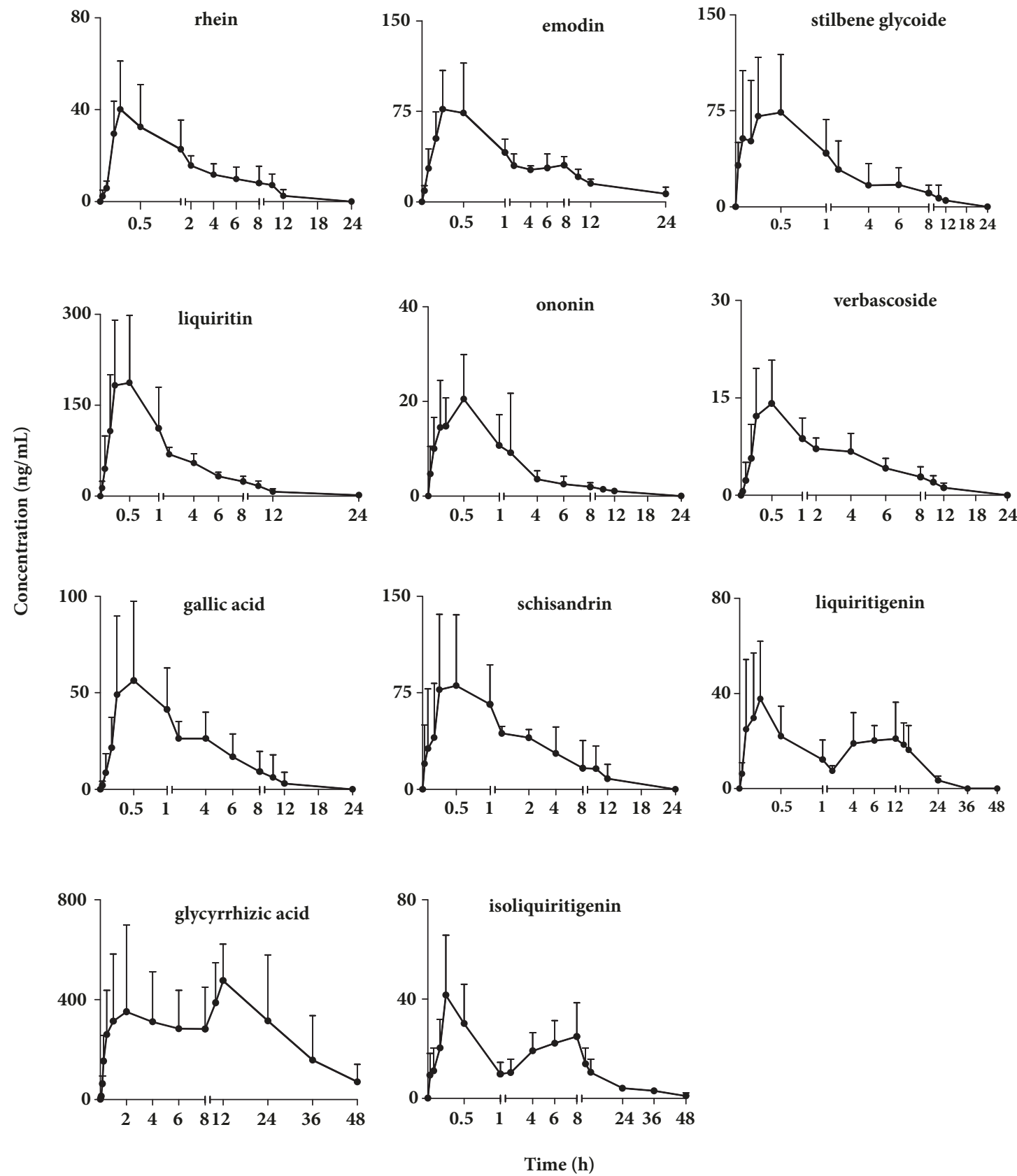

FIGURE 3: Mean plasma concentration-time curves of rhein, emodin, stilbene glycoside, liquiritin, ononin, verbascoside, gallic acid, schisandrin, liquiritigenin, glycyrrhizic acid, and isoliquiritigenin after oral administration of TMYX extract $(\operatorname{mean} \pm \mathrm{SD}, \mathrm{n}=6)$.

indicates that the ingredient has a longer treatment time, especially isoliquiritigenin and glycyrrhizic acid, which can still be detected in vivo at $48 \mathrm{~h}$.

\section{Conclusion}

In our experiment, we developed a method of HPLC-MS/MS for detecting the rhein, emodin, stilbene glycoside, liquiritin, ononin, verbascoside, gallic acid, schisandrin, liquiritigenin, glycyrrhizic acid, and isoliquiritigenin in rat plasma. The pharmacokinetic parameters would be helpful for the further development and usage in clinical of TMYX. The 11 active ingredients of TMYX, which were absorbed into plasma, would provide data support for quality control improvement.

\section{Data Availability}

The data used to support the findings of this study are available from the corresponding author upon request. 


\section{Conflicts of Interest}

The authors declare that there are no conflicts of interest.

\section{Acknowledgments}

This study was supported by National Natural Science Foundation of China (81673824 and 81503457) and Tianjin Municipal Education Commission Research Project (2017KJ139).

\section{References}

[1] P. Shi, X. Lin, and H. Yao, "A comprehensive review of recent studies on pharmacokinetics of traditional Chinese medicines (2014-2017) and perspectives," Drug Metabolism Reviews, vol. 50, no. 2, pp. 161-192, 2017.

[2] Y. Liu, Z. Yang, J. Cheng, and D. Fan, "Barriers and countermeasures in developing traditional Chinese medicine in Europe," Frontiers of Medicine, vol. 10, no. 3, pp. 360-376, 2016.

[3] N.-D. Zhang, T. Han, and B.-K. Huang, "Traditional Chinese Medicine formulas for the treatment of osteoporosis: implication for antiosteoporotic drug discovery," Journal of Ethnopharmacology, vol. 189, pp. 61-80, 2016.

[4] L. Chen, X. Wang, Y. Liu, and X. Di, "Dual-target screening of bioactive components from traditional Chinese medicines by hollow fiber-based ligand fishing combined with liquid chromatography-mass spectrometry," Journal of Pharmaceutical and Biomedical Analysis, vol. 143, pp. 269-276, 2017.

[5] S. Li and B. Zhang, "Traditional Chinese medicine network pharmacology: theory, methodology and application," Chinese Journal of Natural Medicines, vol. 11, no. 2, pp. 110-120, 2013.

[6] X. J. Cai, Y. Wang, L. M. Hu, and Y. C. Jin, "Effect of Tongmai Yangxin pill in treating adrenaline-induced acute arrhythmia," Journal of Tianjin University of Traditional Chinese Medicine, vol. 28, no. 3, pp. 133-135, 2009.

[7] S. Tao, X.-Y. Liang, and Y. Wang, "Screening of active compounds with myocardial protective effects from Tongmai Yangxin pill," Journal of Zhejiang University (Medical Sciences), vol. 44, no. 2, pp. 145-153, 2015.

[8] Y. Fan, S. Man, H. Li, Y. Liu, Z. Liu, and W. Gao, "Analysis of bioactive components and pharmacokinetic study of herb-herb interactions in the traditional Chinese patent medicine Tongmai Yangxin Pill," Journal of Pharmaceutical and Biomedical Analysis, vol. 120, pp. 364-373, 2016.

[9] F. J. Meng and Q. L. Zhan, "Clinical effect of tongmai yangxin pill in treating coronary heart disease patients complicated with angina pectoris and the impact on vascular endothelial function," Practical Journal of Cardiac Cerebral Pneumal and Vascular Disease, vol. 24, no. 5, pp. 84-86, 2016.

[10] L. Li, N. Liu, X. Dai et al., "Development of a dual screening strategy to identify pro-angiogenic compounds from natural products: application on Tongmai Yangxin Pills," RSC Advances, vol. 6, no. 116, pp. 115308-115316, 2016.

[11] N. Liu, L. Li, X. Zhu et al., "A High Content Screening Assay to Identify Compounds with Anti-Epithelial-Mesenchymal Transition Effects from the Chinese Herbal Medicine Tong-MaiYang-Xin-Wan," Molecules, vol. 21, no. 10, p. 1340, 2016.

[12] S. Tao, Y. Huang, Z. Chen, Y. Chen, and Y. Wang, "Rapid identification of anti-inflammatory compounds from Tongmai Yangxin Pills by liquid chromatography with high-resolution mass spectrometry and chemometric analysis," Journal of Separation Science, vol. 38, no. 11, pp. 1881-1893, 2015.

[13] Z. Chen, Studies magnetic beads based approaches for rapid discovery of bioactive compounds from traditional Chinese medicine, Zhejiang University, Hangzhou, China, 2014.

[14] Y. Y. Fan, The Pharmacokinetic Study of Chinese Patent Medicine Tongmai Yangxin Pill, Tianjin University, Tianjin, China, 2016.

[15] M. Xu, G. Wang, H. Xie, Q. Huang, W. Wang, and Y. Jia, "Pharmacokinetic comparisons of schizandrin after oral administration of schizandrin monomer, Fructus Schisandrae aqueous extract and Sheng-Mai-San to rats," Journal of Ethnopharmacology, vol. 115, no. 3, pp. 483-488, 2007.

[16] L. Zhang, W.-F. Ma, J. Li et al., "Influence of processing on pharmacokinetic of typical constituents in radix polygoni multiflori after oral administration by LC-ESI-MS/MS," Journal of Ethnopharmacology, vol. 148, no. 1, pp. 246-253, 2013. 


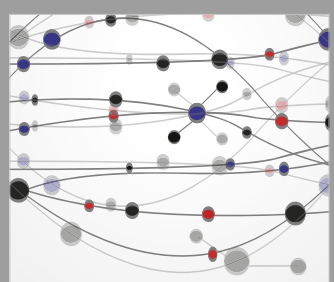

The Scientific World Journal
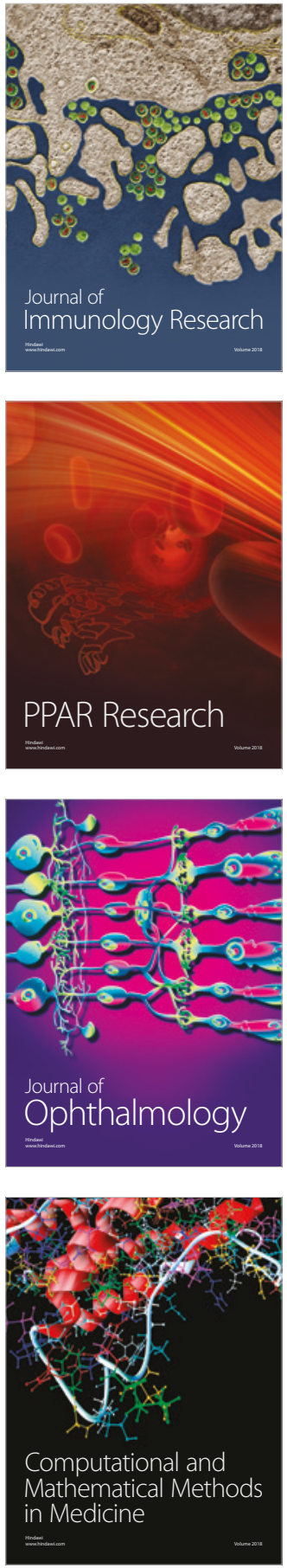

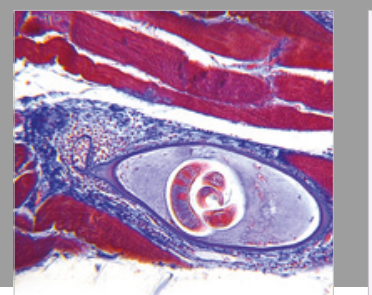

Gastroenterology Research and Practice

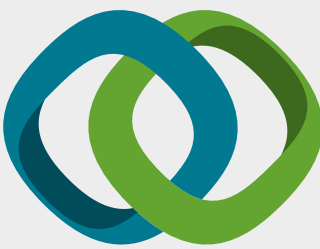

\section{Hindawi}

Submit your manuscripts at

www.hindawi.com
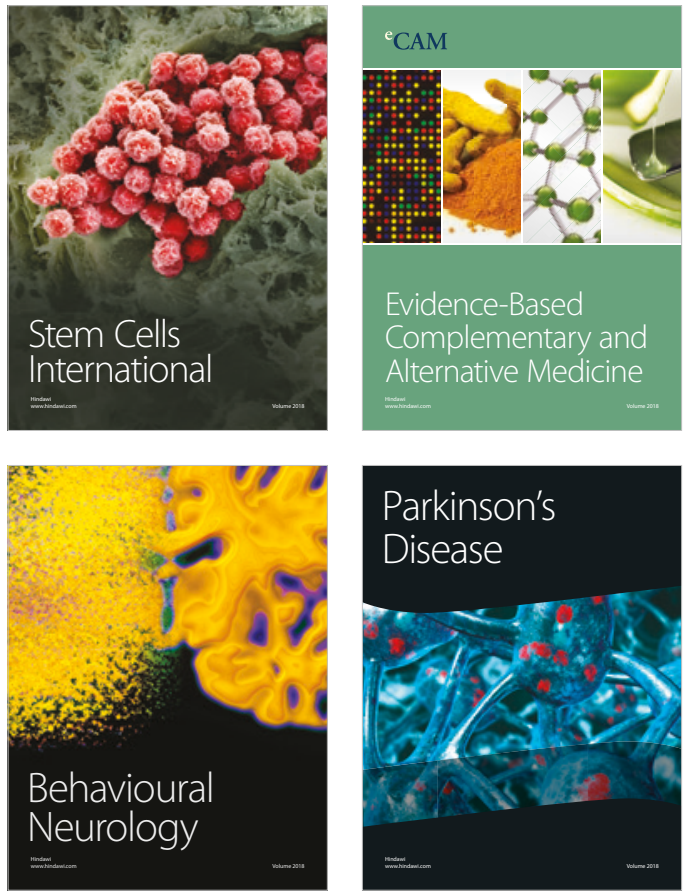

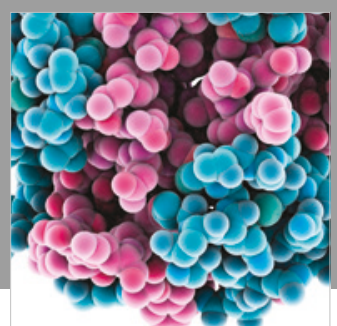

ournal of

Diabetes Research

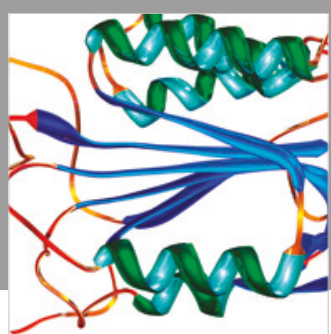

Disease Markers
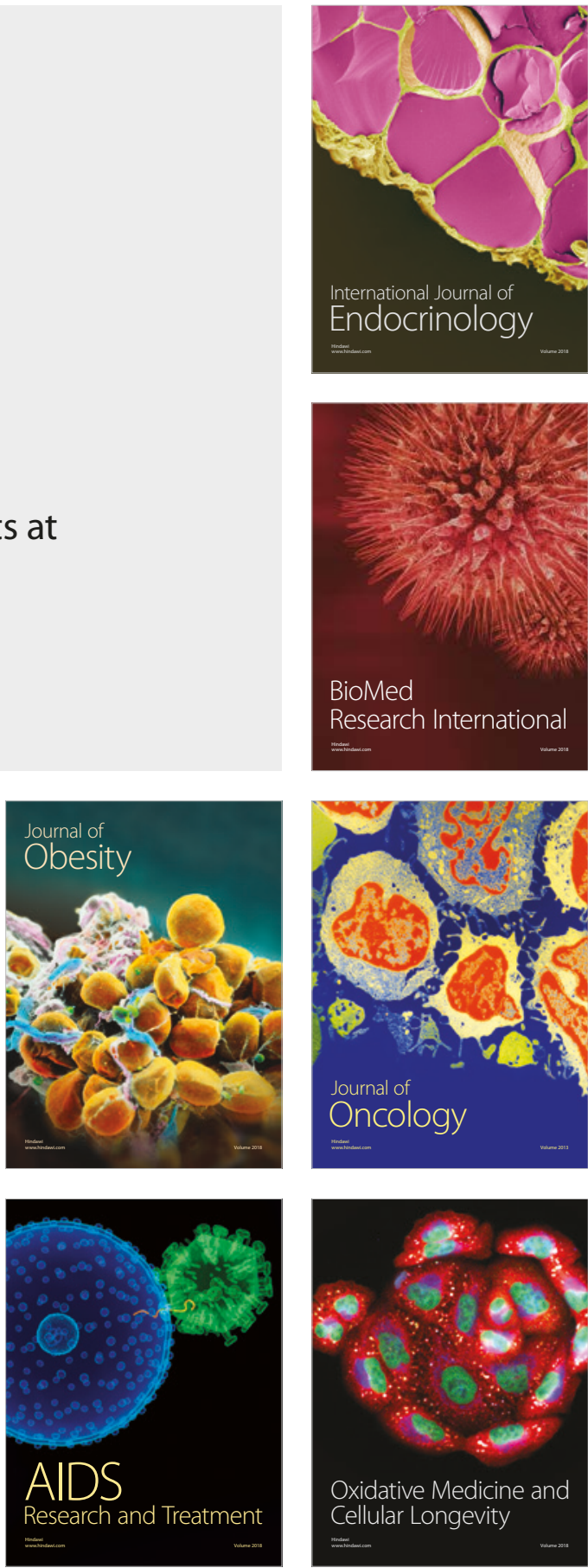